PEREIRA, C.; JUNQUEIRA, A.M.R.; OLIVEIRA, S.A. Balanço nutricional e incidência de queima de bordos em alface produzida em sistema hidropônico - NFT. Horticultura Brasileira, Brasília, v.23, n.3, p.810-814, jul-set 2005.

\title{
Balanço nutricional e incidência de queima de bordos em alface produzi- da em sistema hidropônico - NFT
}

\author{
Claudio Pereira; Ana Maria R. Junqueira; Sebastião Alberto de Oliveira \\ Universidade de Brasília, C. Postal 4.508, 70910-970 Brasília-DF; e-MAIL:anamaria@unb.br
}

\section{RESUMO}

Para avaliar o efeito do balanço nutricional na incidência de queima de bordos em plantas de alface, conduzidas em hidroponia, foi instalado um experimento em área da Universidade de Brasília, de outubro/2001 a junho/2002. Na primeira etapa do experimento, foram adotadas quatro condutividades elétricas $(0,5 ; 1,5 ; 2,5$ e 4,0 $\mathrm{mS} . \mathrm{cm}^{-1}$ ) para avaliar a que apresentaria a maior incidência de queima de bordos. Em uma segunda etapa, adotou-se a concentração de $4,0 \mathrm{mS} . \mathrm{cm}^{-1}$ e aplicações foliares de três fontes de cálcio $\left(\mathrm{CaCl}_{2}\right.$, $\mathrm{CaB}_{2}{ }^{\circledR} \mathrm{e} \mathrm{CaNO}_{3}$ ) com o objetivo de prevenir a ocorrência dos sintomas. Utilizou-se a formulação proposta por Castellane e Araújo em 1995, variando-se a concentração total dos nutrientes para atingir a condutividade elétrica desejada. O delineamento experimental foi em faixas, com quatro tratamentos e três repetições com 25 plantas cada. Foram avaliados a porcentagem de plantas com incidência de queima de bordos, teor foliar de macro e micronutrientes e peso fresco. Utilizou-se o sistema DRIS para análise dos teores foliares de nutrientes. Foi observado que a solução nutritiva de $4 \mathrm{mS} . \mathrm{cm}^{-1}$ apresentou porcentagem de plantas com queima de bordos superior às soluções com concentração de 2,5 e $1,5 \mathrm{mS} . \mathrm{cm}^{-1}$. Porém, não diferiu estatisticamente da solução com $0,5 \mathrm{mS} \cdot \mathrm{cm}^{-1}$. Na segunda etapa, verificou-se que zinco foi o nutriente mais limitante, em função da alta concentração de boro, tendo sido o responsável pela incidência dos sintomas de queima de bordos. Desta forma, as pulverizações com cálcio, independentemente da fonte, não foram eficientes no controle ou redução dos sintomas.

Palavras-chave: Lactuca sativa L., DRIS, análise foliar de nutrientes, balanço nutricional, produção.

\begin{abstract}
Nutrients balance and tip burn incidence in lettuce from hydroponic system - NFT

Aiming to evaluate the effect of nutrients balance in lettuce tip burn incidence, an experiment was carried out in Brasília, Brazil, from October 2001 until June 2002, in two phases. During the first phase four solution concentrations $\left(0.5 ; 1.5 ; 2.5\right.$ and $\left.4.0 \mathrm{mS.cm}{ }^{-1}\right)$ were evaluated to determine the concentration with the highest percentage of tip burn. During the second phase, a solution concentration of 4,0 $\mathrm{mS} . \mathrm{cm}^{-1}$ and foliar applications of three calcium sources $\left(\mathrm{CaCl}_{2}, \mathrm{CaB}_{2}{ }^{\circledR}\right.$ and $\mathrm{CaNO}_{3}$ ) were applied to plants to prevent tip burn. Nutritive solution proposed by Castellane and Araújo in 1995 was used in both phases. The evaluated parameters were percentage of plants with tip burn incidence, leaf nutrient concentration and plant fresh weight. In the first trial the more concentrated $\left(4,0 \mathrm{mS} . \mathrm{cm}^{-1}\right)$ and the less concentrated $\left(0,5 \mathrm{mS} . \mathrm{cm}^{-1}\right)$ nutritive solution presented the highest percentage of plants with the symptoms. In the second trial, the zinc deficiency induced by boron high concentration, was responsible for the symptoms. This was the reason why calcium leaf application was not effective on reducing foliar symptoms.
\end{abstract}

Keywords: Lactuca sativa L., DRIS, leaf nutrients analysis, nutritional balance, production.

(Recebido para publicação em 6 de outubro de 2004 e aceito em 7 de abril de 2005)

$\mathrm{A}$ queima de bordos em alface é uma desordem fisiológica atribuída à deficiência de cálcio nas folhas. Este nutriente é um dos constituintes do pectato de cálcio, composto que atua como elemento cimentante da parede celular e a sua deficiência leva a um enfraquecimento da estrutura e rompimento dos vasos lactíferos, com isso há liberação do látex, levando a um colapso celular e necrose do tecido (FERNANDES; MARTINS, 1999; MORGAN, 2000).

Várias situações são sugeridas na literatura para a ocorrência de queima de bordos, a grande maioria delas associada à deficiência de cálcio, induzida por condições edafoclimáticas adversas do meio: excessiva condutividade elé- trica (CE), normalmente encontrada em condições de manejo inadequado da solução nutritiva; temperatura ambiental e umidade relativa altas (TIBBTIZ; PALZKILL, 1979; BRUUM; SCHENK, 1993; FERNANDES; MARTINS, 1999; MORGAN, 2000).

O sistema DRIS (Diagnosis and Recommendation Integrated System) é utilizado para avaliar os níveis dos nutrientes fornecidos às plantas, bem como as limitações e antagonismo entre nutrientes. O DRIS, proposto por Beaufils (1973), é um sistema de interpretação de resultados de análises de tecidos vegetais. O método compara razões entre pares de nutrientes de uma lavoura amostrada com valores de re- ferência (normas DRIS) de um grupo de produtividade desejada (MALAVOLTA et al., 1997; JÚNIOR et al., 2002) por meio de fórmulas, calculando um índice para cada nutriente envolvido na diagnose.

Podem ser destacadas algumas vantagens do DRIS, em relação a outras diagnoses, no esclarecimento de questões quanto ao equilíbrio nutricional da planta: a escala nutricional fornecida é contínua; os nutrientes são ordenados dos mais deficientes para os que apresentam teores excessivos e, sobretudo, possibilita identificar casos nos quais a produção está sendo limitada por algum desequilíbrio nutricional, mesmo 
quando estes níveis não estão visivelmente críticos (MALAVOLTA et al., 1997; JÚNIOR et al., 2002).

Este trabalho teve como objetivos identificar o nível de concentração da solução nutritiva mais propício à incidência de sintomas de queima de bordos, avaliar a eficiência de três fontes de cálcio como tratamento preventivo/ curativo e, utilizando o sistema DRIS, identificar os elementos limitantes ao desenvolvimento vegetativo das plantas, bem como identificar relações antagônicas entre nutrientes.

\section{MATERIAL E MÉTODOS}

$\mathrm{O}$ experimento foi conduzido em ambiente protegido, utilizando-se uma estufa, modelo arco, coberta com filme de polietileno de 75 micras, com pé direito de 3,0 metros, localizada na $\mathrm{Fa}$ zenda Água Limpa, Universidade de Brasília (DF). O sistema de cultivo utilizado foi o hidropônico, tipo NFT. O experimento foi realizado em duas etapas. O delineamento experimental adotado foi em faixas, com quatro tratamentos, três repetições com 25 plantas cada.

O sistema foi composto por quatro conjuntos de tanques subterrâneos, bancadas e motobombas independentes, possuindo os conjuntos as mesmas dimensões e características técnicas. A vazão adotada foi de $1,5 \mathrm{~L} / \mathrm{min} /$ canaleta, e o ciclo durante o dia foi intermitente, com períodos de 15 minutos circulando a solução nutritiva e 15 minutos parados. Durante a noite, o sistema permaneceu desligado.

Nas duas etapas foi utilizada a cultivar Verônica pelo fato de ser adotada por um número expressivo de produtores hidropônicos da região e apresentar boa resistência ao pendoamento. As mudas foram produzidas em bandejas de isopor com 288 células e substrato comercial $\left(\right.$ Plantmax $\left.^{\circledR}\right)$ e permaneceram em casade-vegetação por cerca de 30 dias após o semeio. Antes do transplantio as raízes foram lavadas em água corrente.

A primeira etapa foi realizada de outubro a dezembro/2001, verão, período em que na região do DF tem sido observada a ocorrência de queima de bordos em alface produzida em sistema hidropônico.
Esta etapa baseou-se na hipótese de que a queima de bordos seria induzida pela excessiva condutividade elétrica da solução, condição normalmente encontrada onde não há manejo adequado da solução nutritiva. Foram avaliados quatro níveis de concentração da solução nutritiva proposta por Castellane e Araújo (1995), adotando-se os seguintes tratamentos: $\mathrm{T} 1=4,0 ; \mathrm{T} 2=2,5 ; \mathrm{T} 3=1,5 \mathrm{e}$ $\mathrm{T} 4=0,5 \mathrm{mS} \cdot \mathrm{cm}^{-1}$. Todas as relações entre nutrientes propostas na solução original foram mantidas, variando tão somente a concentração das mesmas (Tabela 1).

A segunda etapa do experimento foi realizada de abril a junho/2002, utilizando-se a solução nutritiva com concentração de 4,0 mS.cm ${ }^{-1}$. Observou-se que a incidência de sintomas de queima de bordos no tratamento que utilizava a solução mais concentrada $\left(4,0 \mathrm{mS} . \mathrm{cm}^{-1}\right)$ não diferiu estatisticamente da observada a $0,5 \mathrm{mS} \cdot \mathrm{cm}^{-1}$. Porém, como os produtores hidropônicos tendem a utilizar soluções mais concentradas, optou-se pela solução nutritiva de $4,0 \mathrm{mS} . \mathrm{cm}^{-1}$ nesta etapa. Foram avaliadas, então, três fontes de cálcio, aplicadas via pulverização foliar, duas citadas por Resh (1997), cloreto de cálcio $(0,70 \%)$ e nitrato de cálcio $(0,75 \%)$ e uma terceira fonte, atualmente em uso pelos produtores hidropônicos, o $\mathrm{CaB}_{2}{ }^{\circledR}$, como prevenção ou remediação da deficiência de cálcio e queima de bordos. Todas as três fontes possuem concentração de $0,13 \%$ de $\mathrm{Ca}^{2+}$, equivalente à quantidade de cálcio fornecida em uma solução de nitrato de cálcio $0,75 \%$, sendo aplicados $200 \mathrm{ml}$ por bancada. Foram realizadas três pulverizações: aos 10; 17 e 24 dias após o transplantio.

Os tratamentos foram assim distribuídos: $\mathrm{T} 1\left(\mathrm{CaB}_{2}{ }^{\circledR}\right), \mathrm{T} 2$ (testemunha), T3 (nitrato de cálcio) e T4 (cloreto de cálcio). As relações K:N:P:Ca:Mg:S propostas na formulação original foram mantidas, variando tão somente a concentração da solução nutritiva.

Em ambas as etapas, o espaçamento adotado foi $7,5 \times 7,5 \mathrm{~cm}$ nas canaletas de pré-crescimento e $30 \times 30 \mathrm{~cm}$ nas canaletas de terminação. $\mathrm{O}$ pH da solução foi mantido em torno de 6,0 com a utilização de solução de hidróxido de sódio $1 \mathrm{~N}$, para elevação do pH e, poste- rior calibração com peagâmetro. A condutividade elétrica era ajustada no momento de preparo da solução. A proporção volume de solução/número de plantas foi elevada, cerca de 2,66 L.planta ${ }^{-1}$, e foi pequeno o período em que a solução nutritiva permaneceu nos tanques antes da total substituição (cerca de sete dias). Assim, a queda de condutividade elétrica da solução foi mínima.

As plantas permaneceram 30 dias nas bancadas, ao final dos quais avaliouse a porcentagem de plantas com sintomas de queima de bordos por tratamento. Em seguida procedeu-se a colheita de quatro plantas por repetição. Estas plantas foram pesadas e foram avaliados os teores de macro e micronutrientes da parte aérea.

Os dados foram submetidos à análise de variância e as médias comparadas pelo teste de Tukey, ao nível de 5\% de probabilidade. O sistema DRIS foi utilizado para avaliação do balanço dos nutrientes em todos os tratamentos, determinação do elemento mais limitante e a existência de relação antagônica entre nutrientes.

\section{RESULTADOS E DISCUSSÃO}

\section{Porcentagem de queima de bordos}

Na primeira etapa do experimento, a solução nutritiva com condutividade elétrica próxima a $2,5 \mathrm{mS} . \mathrm{cm}^{-1}$ não apresentou incidência de sintomas de queimas de bordos (Tabela 2), concordando com o sugerido por Castellane e Araújo (1995); Faquin et al. (1998) e Furlani (1998) que recomendam como faixa ideal de condutividade elétrica para alface valores entre 2,0 e $3,0 \mathrm{mS} . \mathrm{cm}^{-1}$. A porcentagem de plantas com sintomas de queima de bordos foi semelhante para soluções com 4,0 e $0,5 \mathrm{mS} . \mathrm{cm}^{-1}$, enquanto que a porcentagem de plantas com sintomas na solução com $1,5 \mathrm{mS} . \mathrm{cm}^{-1}$ foi superior à observada na solução com $2,5 \mathrm{mS} . \mathrm{cm}^{-1} \mathrm{e}$ inferior às demais.

Na segunda etapa, o tratamento testemunha, onde não houve aplicação foliar de cálcio, apresentou menor porcentagem de plantas com sintomas de queima de bordos, quando comparado aos tratamentos onde foram aplicados $\mathrm{CaB}_{2}{ }^{\circledR} \mathrm{e} \mathrm{CaCl}_{2}$. Porém, o tratamento tes- 
Tabela 1. Solução original proposta por Castellane e Araújo em 1995, recomendada para alface, e derivadas desta adotadas no cultivo hidropônico de alface cv. Verônica, na primeira etapa do experimento. Brasília, UnB, 2002.

\begin{tabular}{|c|c|c|c|c|c|}
\hline \multirow[b]{2}{*}{ Fonte de nutriente } & \multicolumn{5}{|c|}{ Tratamentos } \\
\hline & $\begin{array}{l}\text { Original } \\
g / 1000 \mathrm{~L}\end{array}$ & $\begin{array}{c}\mathrm{T1} \\
\mathrm{g} / 1000 \mathrm{~L}\end{array}$ & $\begin{array}{c}\mathrm{T} 2 \\
\mathrm{~g} / 1000 \mathrm{~L}\end{array}$ & $\begin{array}{c}\text { T3 } \\
g / 1000 \mathrm{~L}\end{array}$ & $\begin{array}{c}\mathrm{T} 4 \\
\mathrm{~g} / 1000 \mathrm{~L}\end{array}$ \\
\hline Nitrato de Potássio & 954,00 & $1.254,51$ & 785,14 & 471,28 & 157,41 \\
\hline Nitrato de Cálcio & 947,00 & $1.245,31$ & 779,83 & 467,82 & 156,26 \\
\hline Cloreto de Potássio & 123,00 & 161,09 & 100,82 & 60,52 & 20,21 \\
\hline Ácido Fosfórico * & 278,00 & 365,90 & 229,00 & 137,46 & 45,91 \\
\hline Sulfato de Magnésio & 240,00 & 315,60 & 197,52 & 118,56 & 39,60 \\
\hline Ferrilene ** & 83,00 & 108,93 & 68,00 & 24,00 & 13,48 \\
\hline Bórax & 2,85 & 3,75 & 2,35 & 1,41 & 0,47 \\
\hline Sulfato de Cobre & 0,19 & 0,25 & 0,16 & 0,09 & 0,03 \\
\hline $\begin{array}{l}\text { Sulfato Sulfato de } \\
\text { Manganês }\end{array}$ & 1,70 & 2,24 & 1,40 & 0,84 & 0,28 \\
\hline Sulfato de Zinco & 1,15 & 1,51 & 0,95 & 0,57 & 0,19 \\
\hline Molibdato de sódio & 0,12 & 0,16 & 0,10 & 0,06 & 0,02 \\
\hline $\mathrm{CE}^{* * *}$ total em mS.cm-1 & 3,04 & 4,00 & 2,50 & 1,50 & 0,50 \\
\hline
\end{tabular}

*Fonte de fósforo original - MAP. ** Fonte de ferro original - Sulfato Ferroso. *** CE $=$ condutividade elétrica.

temunha não diferiu estatisticamente do tratamento pulverizado com $\mathrm{CaNO}_{3}$. A porcentagem de plantas apresentando sintomas foi significativamente superior nas plantas pulverizadas com $\mathrm{CaB}_{2}{ }^{\circledR} \mathrm{e}$ $\mathrm{CaCl}_{2}$. Observou-se que a aplicação foliar do cálcio não proporcionou resultados positivos e no caso do $\mathrm{CaB}_{2}{ }^{\circledR}$, utilizado pelos produtores hidropônicos da região do DF, a porcentagem de plantas com sintomas aumentou (Tabela 2).

Teor foliar de nutrientes e peso fresco

De acordo com Bruum e Schenk (1993); Huett (1994); Fernandes e Martins (1999) e Morgan (2000), a prin- cipal causa da ocorrência de queima de bordos em alface conduzida em sistema hidropônico é a deficiência de cálcio, ocasionada principalmente por excessiva condutividade elétrica da solução nutritiva. Contudo, observou-se, mediante a análise do teor foliar de nutrientes em ambas as etapas (Tabela 2), não ser possível determinar em função da condutividade elétrica, uma tendência clara de acúmulo ou deficiência de determinado nutriente, incluindo o cálcio.

Não foi observada diferença estatística significativa entre os valores de peso fresco observados nas plantas conduzidas nas soluções com 4,0 e 0,5
$\mathrm{mS} / \mathrm{cm}$ (Tabela 2). As plantas provenientes da solução com 2,5 mS/cm apresentaram maior peso fresco, possivelmente, devido às características iônicas ideais apresentadas pela solução nutritiva, o que proporcionou maior turgescência das plantas, absorção mais adequada de nutrientes e melhor desenvolvimento vegetativo.

Plantas provenientes da solução com $1,5 \mathrm{mS} / \mathrm{cm}$ apresentaram peso fresco intermediário, comparado aos observados nos dois extremos de condutividade elétrica, diferindo estatisticamente dos demais. Condutividade elétrica acima da recomendada, devido à maior pressão osmótica, proporciona menor absorção de água, redução no transporte de nutrientes, menor desenvolvimento e menor ganho de peso. Abaixo do recomendado, a solução possui pressão osmótica menor, a planta absorve mais água, porém o ganho de peso é menor, pois os nutrientes são ofertados em quantidade inadequada à demanda da planta.

Não foi observada diferença estatística significativa para peso fresco entre plantas que receberam pulverização de $\mathrm{CaB}_{2}{ }^{\circledR}, \mathrm{CaNO}_{3}$ e a testemunha. A pulverização de $\mathrm{CaCl}_{2}$ reduziu o peso fresco (Tabela 2), que apresentou média estatisticamente inferior aos demais tratamentos. Condições de alta salinidade impõem às plantas dois fatores a serem superados: pressão osmótica aumentada e potencial hídrico diminuído. Essas altas concentrações levam ao aumento indireto de íons potencialmente tóxicos como o $\mathrm{Na}^{+}$e o $\mathrm{Cl}^{-1}$, combinando estes

Tabela 2. Média dos teores de macro e micronutrientes, queima de bordos e peso fresco de alface, cv. Verônica, em função da condutividade elétrica da solução e adição de cálcio. Brasília, UnB, 2002.

\begin{tabular}{|c|c|c|c|c|c|c|c|c|c|c|c|c|c|}
\hline \multirow{2}{*}{ Tratamentos } & $\mathbf{N}$ & $\mathbf{P}$ & K & $\mathbf{M g}$ & $\mathbf{S}$ & B & $\mathrm{Cu}$ & \multirow{2}{*}{$\begin{array}{c}\mathrm{Fe} \\
\left(\mathrm{mg}^{\mathrm{kg}} \mathbf{k g}^{-1}\right)\end{array}$} & $M n$ & $\mathrm{Zn}$ & \multirow{2}{*}{$\begin{array}{c}\text { Queima } \\
\text { de } \\
\text { bordos } \\
(\%)\end{array}$} & \multirow{2}{*}{$\begin{array}{l}\text { peso } \\
\text { Fresco } \\
\text { (gramas) }\end{array}$} & \multirow{2}{*}{$\begin{array}{c}\mathrm{Ca}^{2+} \\
\left(\mathrm{g} \mathrm{kg}^{-1}\right)\end{array}$} \\
\hline & \multicolumn{7}{|c|}{$\left(\mathbf{g} \cdot \mathrm{kg}^{-1}\right)$} & & & & & & \\
\hline $0,5 \mathrm{mS} \cdot \mathrm{cm}^{-1}$ & 32,2 & 5,6 & 47,5 & 1,7 & 2,4 & 19,3 & 4,3 & 275,0 & 47,0 & 87,6 & $21,32 a^{* *}$ & $211,61 c^{* *}$ & $12,13 a^{* *}$ \\
\hline $1,5 \mathrm{mS} . \mathrm{cm}^{-1}$ & 37,2 & 6,3 & 75,6 & 2,0 & 3,0 & 23,3 & 7,3 & 286,3 & 157,0 & 96,0 & $13,33 b$ & $293,79 b$ & $11,53 a b$ \\
\hline $2,5 \mathrm{mS} . \mathrm{cm}^{-1}$ & 41,4 & 8,5 & 85,9 & 2,0 & 3,0 & 24,6 & 8,3 & 300,0 & 103,6 & 92,0 & $0,00 \mathrm{c}$ & $405,62 \mathrm{a}$ & $11,30 \mathrm{~b}$ \\
\hline $4,0 \mathrm{mS} . \mathrm{cm}^{-1}$ & 47,7 & 8,2 & 76,2 & 2,4 & 4,0 & 27,6 & 11,7 & 414,0 & 116,3 & 124,0 & 18,67 a & $192,85 \mathrm{c}$ & $11,06 \quad b$ \\
\hline 4,0 mS.cm ${ }^{-1}+\mathrm{CaB} 2 \AA$ & 47,5 & 7,5 & 80,0 & 1,8 & 3,1 & 33,6 & 7,3 & 324,0 & 79,3 & 54,0 & 28,0 a & $283,35 \mathrm{a}$ & $12,16 \quad b$ \\
\hline $4,0 \mathrm{mS} . \mathrm{cm}^{-1}$ & 43,9 & 7,3 & 91,5 & 1,9 & 3,4 & 33,3 & 8,0 & 393,0 & 81,0 & 55,3 & $8,0 \mathrm{c}$ & $278,01 \mathrm{a}$ & 16,50 a \\
\hline 4,0 mS.cm ${ }^{-1}+\mathrm{CaNO} 3$ & 43,9 & 5,9 & 68,6 & 1,6 & 2,8 & 30,0 & 6,0 & 234,6 & 66,0 & 48,3 & $12,0 \mathrm{c}$ & 276,40 a & $14,43 a b$ \\
\hline $4,0 \mathrm{mS} \mathrm{cm}^{-1}+\mathrm{CaCl} 2$ & 44,1 & 5,8 & 71,9 & 1,6 & 3,0 & 34,0 & 5,6 & 251,6 & 64,0 & 44,0 & $16,0 \mathrm{~b}$ & $264,42 \mathrm{~b}$ & $15,20 a b$ \\
\hline
\end{tabular}

*Etapa 1, conduzida de outubro a dezembro de 2001 e Etapa 2 conduzida de abril a junho de 2002. **Médias seguidas pela mesma letra na coluna não diferem estatisticamente entre si, pelo teste de Tukey, ao nível de $5 \%$ de probabilidade. Coeficiente de variação (\%):queima de bordos $(6,45)$; peso fresco etapa $1(9,02)$, etapa $2(1,14) ; \mathrm{Ca}^{+2}(10,79)$. 
fatores à adição de $\mathrm{Cl}^{-1}$ presente na solução de $\mathrm{CaCl}_{2}$, o que contribuiu para um desempenho negativo deste tratamento.

\section{Correlações entre índices DRIS}

Foi observada alta correlação negativa (antagonismo) entre os índices DRIS do zinco e boro $(r=-0,96)$, devido a uma inibição ocasionada pelos teores de boro em relação aos teores de zinco, presentes na solução nutritiva (Tabela 3 ).

Em relação ao cálcio, observou-se que o mesmo não possui qualquer relação de antagonismo ou sinergismo com os outros nutrientes e tão pouco é o elemento mais limitante ao alcance da produtividade ideal. Verificou-se, ainda, que o Ca não foi o responsável pelos sintomas de queima de bordos observados nos tratamentos. Zinco foi o elemento mais limitante (47,6\% de ocorrência) em todos os tratamentos (Tabela 4). Os aumentos nos níveis de boro induziram a proporcionais deficiências de zinco. $\mathrm{O}$ zinco atua na produção do triptofano, precursor das auxinas (principalmente ácido indol-acético), fundamentais em

Tabela 3. Matriz de correlação entre índices DRIS de nutrientes amostrados em folhas de alface cv. Verônica. Brasília, UnB, 2002.

\begin{tabular}{cccccccccccc}
\hline & $\mathbf{N}$ & $\mathbf{P}$ & $\mathbf{K}$ & $\mathbf{C a}$ & $\mathbf{M g}$ & $\mathbf{S}$ & $\mathbf{B}$ & $\mathbf{C u}$ & $\mathbf{F e}$ & $\mathbf{M n}$ & $\mathbf{Z n}$ \\
\hline $\mathbf{N}$ & 1,00 & 0,50 & 0,93 & 0,51 & $-0,01$ & 0,87 & 0,95 & 0,65 & $-0,92$ & 0,51 & $-0,87$ \\
$\mathbf{P}$ & & 1,00 & 0,72 & 0,85 & 0,83 & 0,81 & 0,23 & 0,96 & $-0,60$ & 0,87 & $-0,02$ \\
$\mathbf{K}$ & & & 1,00 & 0,79 & 0,31 & 0,98 & 0,78 & 0,84 & $-0,98$ & 0,78 & $-0,69$ \\
$\mathbf{C a}$ & & & & 1,00 & 0,77 & 0,85 & 0,25 & 0,90 & $-0,76$ & 0,99 & $-0,15$ \\
$\mathbf{M g}$ & & & & 1,00 & 0,46 & $-0,31$ & 0,75 & $-0,25$ & 0,79 & 0,47 \\
$\mathbf{S}$ & & & & & 1,00 & 0,69 & 0,92 & $-0,94$ & 0,86 & $-0,56$ \\
$\mathbf{B}$ & & & & & & 1,00 & 0,41 & $-0,80$ & 0,25 & $-0,96$ \\
$\mathbf{C u}$ & & & & & & & 1,00 & $-0,76$ & 0,92 & $-0,21$ \\
$\mathbf{F e}$ & & & & & & & & 1,00 & $-0,75$ & 0,75 \\
$\mathbf{M n}$ & & & & & & & & & 1,00 & $-0,13$ \\
$\mathbf{Z n}$ & & & & & & & & & & 1,00 \\
\hline
\end{tabular}

processos de elongação celular. A sua deficiência causa clorose nas folhas mais velhas, levando a uma posterior necrose do tecido. Em estágios mais avançados, ocasiona senescência prematura e/ou diminuição de tamanho de folhas e produção de plantas anãs (RESH, 1997; FAQUIN, 1998).

Observou-se que as plantas que receberam pulverização de $\mathrm{CaB}_{2}{ }^{\circledR}$ tiveram a incidência de sintomas agravada. $\mathrm{O}$ boro atua na planta formando complexos com estruturas orgânicas, principalmente os carboidratos que posteriormente originam hemiceluloses da parede celular e são precursores de lignina, com função análoga à do cálcio, atuando também na cimentação intercelular. Os sintomas de toxidez são clorose e posterior necrose nas margens de folhas mais velhas (RESH, 1997; FAQUIN, 1998), também facilmente confundidos

Tabela 4. Ordem de limitação dos nutrientes, nos diferentes tratamentos, para alface cv. Verônica, considerando as duas etapas do experimento. Brasília, UnB, 2002.

\begin{tabular}{|c|c|c|c|c|c|c|c|c|c|c|c|c|c|c|c|c|c|c|c|c|c|c|}
\hline \multirow[t]{2}{*}{ Tratamentos } & \multirow{2}{*}{$\begin{array}{l}\operatorname{Rep} \\
1\end{array}$} & \multicolumn{21}{|c|}{ Ordem de Limitação dos Nutrientes } \\
\hline & & $\mathrm{K}$ & $>$ & $\mathrm{N}$ & $>$ & B & $>$ & $\mathrm{S}$ & $>$ & $\mathrm{Cu}$ & $>$ & $P$ & $>$ & $\mathrm{Mn}$ & $>$ & $\mathrm{Ca}$ & $>$ & $\mathrm{Mg}$ & $>$ & $\mathrm{Zn}$ & $>$ & $\mathrm{Fe}$ \\
\hline \multirow[t]{3}{*}{$0,5 \mathrm{mS} \cdot \mathrm{cm}^{-1}$} & 2 & $\mathrm{Cu}$ & $>$ & $\mathrm{K}$ & $>$ & $P$ & $>$ & $\mathrm{Mn}$ & $>$ & $\mathrm{Mg}$ & $>$ & $\mathrm{Ca}$ & $>$ & $S$ & $>$ & $\mathrm{Zn}$ & $>$ & $\mathrm{N}$ & $>$ & B & $>$ & $\mathrm{Fe}$ \\
\hline & 3 & $\mathrm{~K}$ & $>$ & $\mathrm{Ca}$ & $>$ & $S$ & $>$ & $\mathrm{N}$ & $>$ & $\mathrm{Mn}$ & $>$ & $\mathrm{Cu}$ & $>$ & $B$ & $>$ & $\mathrm{P}$ & $>$ & $\mathrm{Mg}$ & $>$ & $\mathrm{Zn}$ & $>$ & $\mathrm{Fe}$ \\
\hline & 1 & $\mathrm{~N}$ & $>$ & $P$ & $>$ & B & $>$ & $\mathrm{K}$ & $>$ & $\mathrm{Fe}$ & $>$ & $\mathrm{Cu}$ & $>$ & $\mathrm{S}$ & $>$ & $\mathrm{Mg}$ & $>$ & $\mathrm{Zn}$ & $>$ & $\mathrm{Ca}$ & $>$ & $\mathrm{Mn}$ \\
\hline \multirow[t]{3}{*}{$1,5 \mathrm{mS} . \mathrm{cm}^{-1}$} & 2 & B & $>$ & $\mathrm{N}$ & $>$ & $\mathrm{P}$ & $>$ & $\mathrm{K}$ & $>$ & $\mathrm{Fe}$ & $>$ & $\mathrm{Cu}$ & $>$ & S & $>$ & $\mathrm{Mg}$ & $>$ & $\mathrm{Zn}$ & $>$ & $\mathrm{Mn}$ & $>$ & $\mathrm{Ca}$ \\
\hline & 3 & $\mathrm{P}$ & $>$ & $\mathrm{Fe}$ & $>$ & K & $=$ & $\mathrm{N}$ & $>$ & $\mathrm{Mg}$ & $>$ & B & $>$ & $\mathrm{Cu}$ & $>$ & $\mathrm{Zn}$ & $>$ & $\mathrm{S}$ & $>$ & $\mathrm{Mn}$ & $=$ & $\mathrm{Ca}$ \\
\hline & 1 & $\mathrm{~N}$ & $>$ & $B$ & $>$ & $\mathrm{S}$ & $>$ & $\mathrm{K}$ & $>$ & $\mathrm{P}$ & $>$ & $\mathrm{Cu}$ & $>$ & $\mathrm{Fe}$ & $>$ & $\mathrm{Mg}$ & $>$ & $\mathrm{Zn}$ & $>$ & $\mathrm{Ca}$ & $>$ & $\mathrm{Mn}$ \\
\hline \multirow[t]{3}{*}{ 2,5 mS.cm-1 } & 2 & $\mathrm{Ca}$ & $=$ & $\mathrm{Mn}$ & $>$ & $\mathrm{Zn}$ & $>$ & $\mathrm{K}$ & $>$ & $\mathrm{Mg}$ & $>$ & B & $>$ & $\mathrm{Cu}$ & $>$ & $\mathrm{Fe}$ & $>$ & $\mathrm{S}$ & $>$ & $\mathrm{P}$ & $>$ & $\mathrm{N}$ \\
\hline & 3 & $\mathrm{Zn}$ & $>$ & $\mathrm{Fe}$ & $>$ & $\mathrm{Mg}$ & $>$ & $\mathrm{Mn}$ & $>$ & $\mathrm{Ca}$ & $>$ & $\mathrm{P}$ & $>$ & $\mathrm{Cu}$ & $>$ & $\mathrm{S}$ & $>$ & $\mathrm{K}$ & $>$ & $\mathrm{N}$ & $>$ & $\mathrm{B}$ \\
\hline & 1 & $\mathrm{Mn}$ & $>$ & $\mathrm{Ca}$ & $=$ & $\mathrm{K}$ & $>$ & $\mathrm{P}$ & $>$ & $\mathrm{Mg}$ & $>$ & $\mathrm{Cu}$ & $>$ & $\mathrm{S}$ & $>$ & $\mathrm{Zn}$ & $>$ & $\mathrm{N}$ & $>$ & $B$ & $>$ & $\mathrm{Fe}$ \\
\hline \multirow[t]{3}{*}{4,0 mS.cm-1 } & 2 & B & $>$ & $\mathrm{K}$ & $>$ & $\mathrm{N}$ & $>$ & $\mathrm{Ca}$ & $>$ & $\mathrm{Fe}$ & $>$ & $\mathrm{Mn}$ & $>$ & $\mathrm{P}$ & $>$ & $\mathrm{S}$ & $>$ & $\mathrm{Mg}$ & $>$ & $\mathrm{Zn}$ & $>$ & $\mathrm{Cu}$ \\
\hline & 3 & $\mathrm{~K}$ & $>$ & B & $>$ & $\mathrm{N}$ & $>$ & $\mathrm{P}$ & $>$ & $\mathrm{S}$ & $>$ & $\mathrm{Cu}$ & $>$ & $\mathrm{Ca}$ & $>$ & $\mathrm{Mn}$ & $>$ & $\mathrm{Mg}$ & $>$ & $\mathrm{Fe}$ & $>$ & $\mathrm{Zn}$ \\
\hline & 1 & $\mathrm{Zn}$ & $>$ & $\mathrm{Ca}$ & $>$ & $\mathrm{Mg}$ & $>$ & $\mathrm{Mn}$ & $>$ & $\mathrm{Fe}$ & $>$ & $\mathrm{P}$ & $>$ & $\mathrm{Cu}$ & $>$ & $\mathrm{S}$ & $>$ & $\mathrm{K}$ & $>$ & $\mathrm{N}$ & $>$ & $B$ \\
\hline \multirow[t]{3}{*}{ 4,0 mS.cm ${ }^{-1}+\mathrm{CaB}_{2}{ }^{\circledR}$} & 2 & $\mathrm{Zn}$ & $>$ & $\mathrm{Mg}$ & $>$ & $\mathrm{Ca}$ & $>$ & $\mathrm{Mn}$ & $>$ & $\mathrm{P}$ & $>$ & $\mathrm{Cu}$ & $>$ & $\mathrm{S}$ & $>$ & $\mathrm{K}$ & $>$ & $\mathrm{Fe}$ & $>$ & $\mathrm{N}$ & $>$ & $B$ \\
\hline & 3 & $\mathrm{Ca}$ & $>$ & $\mathrm{Zn}$ & $>$ & $\mathrm{Mn}$ & $=$ & $\mathrm{Mg}$ & $>$ & $\mathrm{P}$ & $>$ & $\mathrm{Cu}$ & $>$ & $\mathrm{S}$ & $>$ & $\mathrm{K}$ & $>$ & $\mathrm{Fe}$ & $>$ & $\mathrm{N}$ & $>$ & $\mathrm{B}$ \\
\hline & 1 & $\mathrm{Zn}$ & $>$ & $\mathrm{Ca}$ & $>$ & $\mathrm{Mg}$ & $>$ & $\mathrm{Mn}$ & $>$ & $\mathrm{P}$ & $>$ & $\mathrm{Cu}$ & $>$ & $\mathrm{K}$ & $>$ & $\mathrm{S}$ & $>$ & $\mathrm{Fe}$ & $>$ & $\mathrm{N}$ & $>$ & $B$ \\
\hline \multirow[t]{3}{*}{ 4,0 mS.cm-1 } & 2 & $\mathrm{Zn}$ & $>$ & $\mathrm{Mg}$ & $>$ & $\mathrm{Ca}$ & $>$ & $\mathrm{Mn}$ & $>$ & $\mathrm{P}$ & $>$ & $\mathrm{Cu}$ & $>$ & $\mathrm{S}$ & $>$ & $\mathrm{K}$ & $>$ & $\mathrm{Fe}$ & $>$ & $\mathrm{N}$ & $>$ & $B$ \\
\hline & 3 & $\mathrm{Ca}$ & $>$ & $\mathrm{Mn}$ & $>$ & $\mathrm{Zn}$ & $>$ & $\mathrm{Mg}$ & $>$ & $\mathrm{P}$ & $>$ & $\mathrm{Cu}$ & $>$ & $\mathrm{S}$ & $>$ & K & $>$ & $\mathrm{N}$ & $>$ & $\mathrm{Fe}$ & $>$ & $B$ \\
\hline & 1 & $\mathrm{Zn}$ & $>$ & $\mathrm{Mg}$ & $>$ & $\mathrm{Ca}$ & $>$ & $\mathrm{Mn}$ & $>$ & $P$ & $>$ & $\mathrm{Cu}$ & $>$ & $\mathrm{S}$ & $>$ & $\mathrm{K}$ & $>$ & $\mathrm{Fe}$ & $>$ & $\mathrm{N}$ & $>$ & $B$ \\
\hline \multirow[t]{3}{*}{$4,0 \mathrm{mS} \cdot \mathrm{cm}^{-1}+\mathrm{CaNO}_{3}$} & 2 & $\mathrm{Zn}$ & $>$ & $\mathrm{Mg}$ & $>$ & $\mathrm{P}$ & $>$ & $\mathrm{Ca}$ & $>$ & $\mathrm{Mn}$ & $>$ & $\mathrm{Cu}$ & $>$ & $\mathrm{Fe}$ & $>$ & $\mathrm{K}$ & $>$ & $\mathrm{S}$ & $>$ & $\mathrm{N}$ & $>$ & $B$ \\
\hline & 3 & $\mathrm{Zn}$ & $>$ & $\mathrm{Mg}$ & $>$ & $\mathrm{Ca}$ & $>$ & $\mathrm{Mn}$ & $>$ & $\mathrm{P}$ & $>$ & $\mathrm{Cu}$ & $>$ & $\mathrm{Fe}$ & $>$ & $\mathrm{S}$ & $>$ & $\mathrm{K}$ & $>$ & $\mathrm{N}$ & $>$ & $\mathrm{B}$ \\
\hline & 1 & $\mathrm{Zn}$ & $>$ & $\mathrm{Mg}$ & $>$ & $\mathrm{P}$ & $>$ & $\mathrm{Ca}$ & $>$ & $\mathrm{Mn}$ & $>$ & $\mathrm{Cu}$ & $>$ & $\mathrm{Fe}$ & $>$ & $\mathrm{S}$ & $>$ & $\mathrm{K}$ & $>$ & $\mathrm{N}$ & $>$ & $B$ \\
\hline \multirow[t]{2}{*}{$4,0 \mathrm{mS} \cdot \mathrm{cm}^{-1}+\mathrm{CaCl}_{2}$} & 2 & $\mathrm{Zn}$ & $>$ & $\mathrm{Mg}$ & $>$ & $\mathrm{Ca}$ & $>$ & $\mathrm{Mn}$ & $>$ & $P$ & $>$ & $\mathrm{Cu}$ & $>$ & $\mathrm{K}$ & $>$ & $\mathrm{S}$ & $>$ & $\mathrm{Fe}$ & $>$ & $\mathrm{N}$ & $>$ & $B$ \\
\hline & 3 & $\mathrm{Zn}$ & $>$ & $\mathrm{Mg}$ & $>$ & $\mathrm{Mn}$ & $>$ & $\mathrm{Ca}$ & $>$ & $P$ & $>$ & $\mathrm{Cu}$ & $>$ & $S$ & $>$ & $\mathrm{Fe}$ & $>$ & $\mathrm{K}$ & $>$ & $\mathrm{N}$ & $>$ & B \\
\hline
\end{tabular}


com a queima de bordos ocasionada pela deficiência de cálcio. Portanto, neste tratamento, verificou-se além da deficiência induzida do zinco, uma provável toxidez por boro.

Os sintomas observados, em ambas as etapas, não apresentaram indícios, de acordo com as concentrações dos níveis nutricionais dos tecidos foliares amostrados, de uma visível deficiência do nutriente cálcio que pudesse responsabilizá-lo pelos sintomas detectados. A análise dos índices DRIS indicou que o cálcio, em todas as suas combinações, não foi o nutriente limitante. De fato, os teores de boro presentes nas soluções nutritivas induziram a uma deficiência de zinco e esse nutriente foi o responsável pelos sintomas de queima de bordos nas plantas em condições de desequilíbrio de condutividade elétrica.

Como normalmente a queima é associada à deficiência de cálcio, quando foram utilizados produtos à base de cálcio, não foi verificado efeito positivo da adição foliar desse nutriente na redução dos sintomas.

\section{AGRADECIMENTOS}

Os autores agradecem ao CNPq, pela concessão de bolsa ao primeiro autor; ao NUCOMP-FAV-UnB e Fazenda Água Limpa, pelo apoio logístico e ao engenheiro agrônomo Michael Quadros, pelo auxílio nos trabalhos de campo.

\section{LITERATURA CITADA}

BEAUFILS, E.R. Diagnosis and recommendation integrated system (DRIS). A General Scheme for experimentation and calibration based on principles developed from research in plant nutrition. Soil Science Bulletin, Pietermaritzburg, v.1, n.1, 132 p. 1973.

BRUUM, I.; SCHENK, M. Influence of nitrogen supply on the occurrence of calcium deficiency in field grown lettuce. Acta Horticulturae, n.339, p.125-136, 1993.

CASTELLANE, P.D.; ARAÚJO, J.A.C. Cultivo sem solo: hidroponia. Jaboticabal: FUNEP, 1995. 43 p.

FAQUIN, V., NETO, A.E.F; VILELA, L.A.A. Produção de alface em hidroponia. Lavras. FAEPE, 1998. 50 p.

FERNANDES, H.S.; MARTINS, S.R. Cultivo de alface em solo em ambiente protegido. Informe Agropecuário, Belo Horizonte, v.20, n.200/201, p.56-63, 1999.
FURLANI, P.R. Instruções para o cultivo de hortaliças de folhas pela técnica de Hidroponia - NFT. Campinas: IAC, 1998. 30 p. (Instituto Agronômico, Boletim Técnico, 168)

HUETT, D.O. Growth, nutrient uptake and tipburn severity of hydroponic lettuce in response to electrical conductivity and $\mathrm{K}: \mathrm{Ca}$ ratio in solution. Australian Journal of Agricultural Research, v.45, n.1, p.251-267, 1994.

JÚNIOR, R.A.R.; CORRÊA, J.B.; CARVALHO, J.G.; GUIMARÃES, P.T.G. Estabelecimento de normas DRIS para o cafeeiro no Sul de Minas Gerais: $1^{\mathrm{a}}$ Aproximação. Ciência Agrotécnica, Lavras, v.26, n.2, p.269-282, 2002.

MALAVOLTA, E.; VITTI, G.C.; OLIVEIRA, S.A. Avaliação do estado nutricional das plantas: princípios e aplicações. $2^{\mathrm{a}}$.ed. Piracicaba: POTAFOS, 319 p.1997.

MORGAN, L. El cálcio: Su Importancia en hidroponia. Boletín Informativo Numero 6 . Universidad Nacional Agraria La Molina , 2000. RESH, H.M. Cultivos hidroponicos: Nuevas técnicas de producción. Mundi-prensa: Espanha, 1997, 509 p.

TIBBITZ, T.W.; PALZKILL, D.A. Requeriment for root-pressure flow to provide adequate calcium to low transpiring tissue. Comm. Soil Sci. and Plat Anal. v.10, p.251-257. 1979. 